\title{
Polymorphisms of Catechol-O-Methyl Transferase (COMT) Gene in Vulnerability to Levodopa-Induced Dyskinesia
}

Svetlana A. Ivanova, ${ }^{1,2}$ Valentina M. Alifirova, ${ }^{3}$ Ivan V. Pozhidaev, ${ }^{1,4}$ Maxim B. Freidin,,${ }^{5,6}$ Irina A. Zhukova, ${ }^{3}$ Diana Z. Osmanova, ${ }^{1,4}$ Natalia G. Zhukova, ${ }^{3}$ Yulia A. Mironova, ${ }^{3}$ Vladimir V. Tiguntsev, ${ }^{1}$ Olga Yu. Fedorenko, ${ }^{1,2}$ Nikolay A. Bokhan, ${ }^{1,3,4}$ Bob Wilffert, ${ }^{7,8}$ Anton J.M. Loonen ${ }^{7,9}$

\begin{abstract}
${ }^{1}$ Mental Health Research Institute, Tomsk National Research Medical Center of the Russian Academy of Sciences, Tomsk, Russian Federation; ${ }^{2}$ National Research Tomsk Polytechnic University, Tomsk, Russian Federation; ${ }^{3}$ Siberian State Medical University, Tomsk, Russian Federation; ${ }^{4}$ National Research Tomsk State University, Tomsk, Russian Federation; ${ }^{5}$ Department of Twin Research and Genetic Epidemiology, King's College London, London, United Kingdom; ${ }^{6}$ Research Institute of Medical Genetics, Tomsk National Research Medical Center of the Russian Academy of Sciences, Tomsk, Russian Federation; ${ }^{7}$ University of Groningen, Groningen Research Institute of Pharmacy, Unit of Pharmacotherapy, Epidemiology and Economics, Groningen, the Netherlands; ${ }^{8}$ University of Groningen, University Medical Center Groningen, Department of Clinical Pharmacy and Pharmacology, Groningen, the Netherlands; ${ }^{9}$ Mental health institute GGZ WNB, Bergen op Zoom, The Netherlands
\end{abstract}

Received, April 16, 2018; Revised, May 21, 2018; Accepted July 31, 2018; Published, August 3, 2018.

ABSTRACT - Purpose. Parkinson's disease (PD), a common neurodegenerative disorder, is usually treated with Levodopa (L-DOPA). The use of this drug, however, is severely limited by the development of side effects of the motor system: Levodopa-induced dyskinesia (LID). The aim of this study is to investigate the association between seven COMT gene single-nucleotide polymorphisms (SNPs) and the development of LID in patients with PD. Methods. 232 Caucasian patients with PD were investigated. 212 patients with PD received Levodopa therapy. Dyskinesia was assessed with the use of the Abnormal Involuntary Movement Scale (AIMS). Genotyping was carried out on seven SNPs of the COMT gene (rs4680, rs6269, rs4633, rs4818, rs769224, rs165774, rs174696) using a real-time PCR method, and blind to the clinical status of the subjects. Results. We found association between four SNPs, rs165774, rs4818, rs4633, rs4680, and LID. When the duration of disease was added as a covariate in regression analysis, however, the results did not reach statistical significance. Only the additive model for rs 165774 was found to be close to be statistical significance $(\mathrm{OR}=$ 1.627 [0.976-2.741], permutation $p=0.057$ ). Conclusions. The results failed to clearly support a contribution of the studied polymorphisms; this may be related to a dominant relationship with the disease duration confounding the effect on the prevalence of LID.

\section{INTRODUCTION}

Over 55 years after the discovery of its beneficial effects, ${ }^{1,2}$ Levodopa remains the mainstay of treatment for Parkinson's disease (PD). The use of Levodopa for the treatment of motor symptoms at all stages of PD is supported by strong evidence. ${ }^{3}$ Longterm treatment with Levodopa for PD, however, is frequently complicated by motor fluctuations and dyskinesias. ${ }^{3-6}$ Monotherapy with dopamine agonists in the early phases of the disease does reduce the risk for dyskinesias compared with the use of Levodopa, but dopamine agonists are unable to prevent dyskinesias once Levodopa is added, and which is always required once disease severity progresses. ${ }^{7}$ Chronic Levodopa treatment has been reported to result in LID in up to $45 \%$ of Levodopa users within five years. ${ }^{8}$ Clinical heterogeneity of LID suggests a significant role of endogenous factors in determining their prevalence, although no effective drug treatment of levodopa-induced dyskinesia (LID) has yet been developed. ${ }^{9}$ Several theories have attempted to explain the pathophysiology of this treatment complication with an ultimate goal to develop such treatments ${ }^{9-11}$, but the exact pathological mechanism has also not yet been elucidated. Recently, Ivanova and Loonen developed the hypothesis that LID is related to an increased vulnerability to excitotoxicity of indirect pathway striatal medium spiny neurons potentiated due to the contribution of increased intracellular oxidative stress. ${ }^{12}$ This last phenomenon is expected to be caused by the same genetic composition which causes the degeneration of dopaminergic nigrostriatal neurons in patients with PD.

Corresponding author: Prof. Anton J.M. Loonen, University of Groningen, Groningen Research Institute of Pharmacy, Antonius Deusinglaan 1, 9713AV Groningen, The Netherlands, Tel: +31 50363 7576, Fax: + 3150363 2772, Email: a.j.m.loonen@,rug.nl 
Abbreviations: COMT, catechol-O-methyltransferase; LID, levodopa-induced dyskinesia; MB-COMT membrane-bound form of COMT; PD Parkinson's disease; PFC, prefrontal cortex; SNP single-nucleotide polymorphism; S-COMT, soluble form of COMT.

Dopamine can be considered to be a particularly neurotoxic endogenous substance: dopamine metabolism results in the release of hydrogen peroxide, which, in turn results in the production of free radicals, which then cause cell damage. ${ }^{13}$ This damage is limited by the influence of certain enzymes such as manganese superoxidedismutase (MnSOD), which scavenge these free radicals. ${ }^{14}$ This neurotoxicity is expected to be potentiated by biological factors which increase the intracellular levels of dopamine.

Catechol-O-methyltransferase (COMT; EC 2.1.1.6) is one of the most important enzymes in levodopa metabolism. It was identified by Julius Axelrod at the National Institute of Mental Health (USA) in the second half of the 1950s. ${ }^{15,16}$ COMT catalyzes methyl group transfer from S-adenosyl-Lmethionine to one of the hydroxyl groups of catechol in the presence of $\mathrm{Mg} 2+$ ions. COMT substrates include a wide variety of catechols (catecholamines, their hydroxylated metabolites, catecholestrogens, ascorbic acid, dietary phytochemicals, and medicinal compounds). ${ }^{15,16}$ The major physiological role of COMT is the elimination of biologically active or toxic catechols. Of special importance is the methylation of Levodopa to 3-O-methyldopa in Levodopa/aromatic amino acid decarboxylase inhibitor-treated Parkinson's disease (PD) patients, because this metabolism markedly limits the availability of Levodopa to the brain. ${ }^{15}$ It has also been suggested that COMT plays a relevant role in modulating prefrontal dopamine neurotransmission. ${ }^{17}$ Polymorphic variants of the COMT gene determine the activity level of this enzyme. ${ }^{17-19}$ COMT exists in two forms: the soluble form (S-COMT) which is located in cytosol, and the membrane-bound form (MB-COMT) which is anchored to the rough endoplasmic reticulum. The two forms differ only by a 50 residue long extension in the MB-form, which is the signal sequence for membrane anchoring. ${ }^{20,21}$ In humans, the COMT gene is located on chromosome 22 band q11.21, and is composed of six exons. ${ }^{15}$ The first two exons are noncoding, while the translation initiation codons for the membrane bound and soluble isoforms are located on the third exon. Two separate promoters direct the synthesis of two partially overlapped transcripts: one of $1.5 \mathrm{~kb}$ that is constitutively expressed, and another of $1.3 \mathrm{~kb}$ which is subject to tissue-specific transcription regulation. The short transcript translates S-COMT and the longer transcript translates MB-COMT, and also the soluble form by the leaky scanning mechanism of translational initiation. ${ }^{15}$ The long transcript has been found in all tissues analyzed. The short transcript, on the other hand, is only found in very small amounts within the human brain. There is, however, no direct correlation between transcript and protein levels. In the human brain S-COMT represents about $30 \%$ of the total COMT. ${ }^{15}$

Although several polymorphisms have been reported for the COMT gene, most of them appear not to have any physiological significance. However, the genetic variant Val158Met (rs4680) with low thermal stability and low COMT activity is a wellestablished polymorphism that could contribute to various neuropsychiatric manifestations. ${ }^{18,19}$ Low COMT activity appears to have predominantly clinical effects within the prefrontal cortex (PFC) as in the PFC DA-transporters are expressed in low abundance within synapses, contrary to, for example, the striatum. ${ }^{19,22}$ Furthermore, the nonsynonymous Ala72Ser (rs6267) polymorphism has been associated with reduced COMT enzyme activity and with a risk for schizophrenia ${ }^{23}$ Apart from the above mentioned Val158Met (rs4680), three singlenucleotide polymorphisms (SNPs) were found: one in the S-COMT promoter region (rs6296) and two in the S- and MB-COMT coding region (c.186C $>\mathrm{T}$, p.62His (rs4633), c.408C $>\mathrm{T}, \mathrm{p} .136 \mathrm{Lys}$ ( $\mathrm{rs} 4818$ ), next to rs4680). These were associated with differences in pain sensitivity and the likelihood of developing a chronic musculoskeletal pain condition. ${ }^{24}$ It was shown that these haplotypes modulate protein expression by altering mRNA secondary structure, thus stressing the functional significance of synonymous variations and importance of haplotypes. ${ }^{18}$ Another relevant synonymous variation includes c.597G $>$ A, p.199Pro (rs769224).$^{18} \mathrm{Xu}$ et al demonstrated an association of the $\mathrm{G}-\mathrm{G}-\mathrm{G}$ vs. A-G-G haplotype of rs 4633 , rs 4818 and rs769224 concerning response to non-SSRIs in major depressive disorder. ${ }^{25}$ Recently published results revealed that homozygosity for rs4633 (TT), rs4680 (AA) and of the two linked rs4633-rs4680 (TT/AA) was significantly associated to Levodopa effects in PD patients, while no significant differences were observed in patients who carried individual rs6269 and rs4818, the two linked rs6269rs4818 and the four combined COMT SNPs. ${ }^{26}$ These last observations contrast to the findings in patients with Attention-Deficit/Hyperactivity Disorder (ADHD) (64\% using medication for it) in who the c.$98 \mathrm{~A}>\mathrm{G}$ (rs6269) was associated with the largest influence on the hyperactivity/impulsivity scores. In 
PD patients, any existing significant differences were found for individual SNPs (rs6269; rs4633; rs4818; and rs4680) in the allele and genotype frequencies between PD cases and controls, but their division into haplotypes with low, medium and high COMT activity revealed that the high activity haplotypes needed a significantly higher levodopa dosage. ${ }^{27}$ In their study, the COMT haplotype seemed to have little influence on the development of Levodopa-induced dyskinesias. Meloto et al identified a functional marker situated in the 39 untranslated region of a newfound splice variant (c.615+739G $>$ A; rs165774) which displays unique substrate specificity, exhibiting enzymatic activity with dopamine, but not with epinephrine. ${ }^{28}$ They established that the pain-protective A allele of rs165774 coincides with lower COMT activity due to increased dopaminergic tone. Associations of rs165774 were also found by Higashiyama et al in patients with schizophrenia vs. healthy controls and by Seib et al in older women with depression. ${ }^{29,30}$ Ittiwut et al found an association between rs4680 and c.615+1354C $>\mathrm{T}$ (rs174696) in European American patients with cocaine-induced paranoia. ${ }^{31}$

In our current study we have investigated a possible existence of an association between rs 4680 , rs6269, rs4633, rs4818, rs769224, rs165774, as well as rs174696 and levodopa-induced dyskinesia in 232 Caucasian PD patients from the neurological department of the Siberian State University Hospital of Tomsk, Russian Federation. The rationale for our study was that these polymorphisms have been demonstrated to induce certain functional changes. It was hypothesized that at least some of the biochemical alterations causing these functional changes might also affect the intracellular dopamine concentration, which could result in changed vulnerability for developing levodopa-induced dyskinesia.

\section{METHODS}

\section{Patients}

The work described in this article was carried out in accordance with The Code of Ethics of the World Medical Association (Declaration of Helsinki 1975, revised in Fortaleza, Brazil, 2013) for experiments involving humans. Patients were recruited from the neurology department of the Siberian State Medical University Hospital in Tomsk, Russian Federation. Written informed consent was obtained from each patient after obtaining approval for the study (protocol N 3604 10.02.2014) from the Local Bioethics Committee of the Siberian State Medical University. None of the participants had a compromised capacity/ability to consent; therefore consent from the next-of-kin was not necessary, and was not recommended by the local ethics committee. The inclusion criteria were a clinical diagnosis of Parkinson's disease according to ICD-10 (G-20) and also prior long-term use of Levodopa. Exclusion criteria were non-Caucasian physical appearance (e.g., Mongoloid, Buryats or Khakassians), relevant psychiatric symptoms or any organic brain disorders other than Parkinson's disease. These patients had not been treated with dopamine antagonists (antipsychotic or antiemetic drugs, including clozapine) for at least three years. Patients were assessed for the presence or absence of dyskinesia during 'on'-phase according to the abnormal involuntary movement scale (AIMS). ${ }^{32-34}$ The AIMS scores were transformed into a binary form of LID (presence or absence of dyskinesia) with Schooler and Kane's criteria. ${ }^{35}$

\section{DNA analysis}

DNA was isolated from the leukocytes in whole peripheral blood using the standard phenolchloroform micro method. ${ }^{36}$ Genotyping was carried out on the MassARRAY Analyzer 4 (Agena Bioscience) using the set SEQUENOM Consumables iPLEX Gold 384. DNA sample preparation for SEQUENOM MassARRAY Analyzer 4 includes several steps: a standard PCR reaction to obtain the amplification products, a shrimp alkaline phosphatase reaction to neutralize the unincorporated dNTPs in the amplification products, the PCR iPLEX Gold extension reaction, and then placing the samples on a special chip (SpectroCHIP array) using Nanodispenser RS1000 prior to loading them into the analyser. Genotyping was carried out on 7 SNPs of COMT genes (rs4680, rs6269, rs4633, rs4818, rs769224, rs165774, rs174696), which were selected after reviewing the literature for possible relevance.

\section{Statistical analysis}

Statistical analyses were performed using SPSS software for Windows, release 17. The MannWhitney test (MWT) was used to compare qualitative traits and $\chi^{2}$ test for categorical traits. The Hardy-Weinberg equilibrium (HWE) of genotypic frequencies was tested using the $\chi^{2}$ test. The $\chi^{2}$ test and the Fisher's exact test, if necessary, were used for between-group comparisons of genotype or allele frequencies.

The analysis of association between the SNPs and the phenotype was carried out using logistic regression with adjustment for covariates. Additive, dominant and recessive models were tested, and the optimum model has been chosen 
using Akaike Information Criterion (AIC). Experiment-wise permutations were used to address the multiple testing issue, hence Bonferroni correction was not applicable. Models with permutation $\mathrm{p}$-value $<0.05$ were considered statistically significant. Odds ratio (OR) and 95\% confidence intervals were estimated to assess the strength of genetic effect. The following filters were applied to the SNPs: minor allele frequency, 5\%; deviation from Hardy-Weinberg equilibrium, $\mathrm{p}=0.007$ (assuming $\alpha=0.05$ for 7 SNPs).

\section{RESULTS}

A total of 232 Caucasian patients with PD were included, consisting of 149 females and 83 males (age range from 40 to 86 years, average age $68.7 \pm 7.6$ years). These patients demonstrated typical PD demographics, with a mean age of onset of $59.0 \pm 9.5$ years, and a mean disease duration of $9.8 \pm 5.6$ years. The distribution of genotypes of studied genes corresponds to the Hardy-Weinberg equilibrium (data not shown). According to the predefined criteria, 58 patients suffered from dyskinesia. We found that four out of seven tested SNPs passed the significance thresholds for association with LID. Since associations can in part be explained by the effects of additional factors, such as sex and age, we carried out a regression analysis using LID as the dependent variable, the genotypes as the fixed factor and sex and age as covariates. Using linear regression models, we found association between four SNPs, rs165774, rs4818, rs4633, and rs4680, and LID; the best model (according to AIC values) was additive for rs 165774 and rs4818 and dominant for rs4633 and rs4680 (Table 1). In case of rs165774, the rare allele was associated with an increased risk of LID (OR $=1.75$ [95\% CI 1.14-2.72]), while in case of rs4818, rs4633 and rs4680, the rare allele or homozygote genotype for the rare allele were protective against LID $(\mathrm{OR}=0.57$ [0.34-0.92], 0.45 [0.23-0.89] and 0.46 [0.23-0.91], respectively). However, when the duration of disease was added as a covariate in regression analysis, the results did not reach statistical significance. Only the additive model for rs165774 was found to be close to statistical significance $(\mathrm{OR}=1.627$ [0.976-2.741], permutation $\mathrm{p}=0.057$ ).

\section{DISCUSSION}

We have failed in this study of 232 white patients with Parkinson's Disease (PD) to establish a clear relationship between any of the seven polymorphisms of the COMT gene and the prevalence of Levodopa-induced dyskinesia (LID).
The associations that were found lost their statistical significance after correcting for disease duration. This last observation may correspond with the previously described association between some COMT gene polymorphisms and the onset of disease in 143 patients with $\mathrm{PD} .{ }^{26}$ Watanabe et al found a slightly higher prevalence of PD in 30/118 patients with homozygosity for the low-activity allele of Val158Met (rs4680), ${ }^{37}$ but these findings were not reproduced by Contin et al in 104 patients, and by Cheshire et al in 285 patients. ${ }^{38,39}$ We had observed previously a significant association with PD for the c.615+739G $>$ A (rs165774), but not Val158Met (rs4680), polymorphism and PD of the same patient population vs. 127 healthy controls. ${ }^{40}$ De Lau et al described the existence of a prospectively assessed correlation between the A-allele of the COMT Val158Met polymorphism and an increased risk of developing dyskinesias in 219 patients with $\mathrm{PD} .{ }^{41} \mathrm{~A}$ comprehensive meta-analysis in which a total of 363 datasets were included, consisting 56,998 cases and 74,668 healthy controls from case control studies, as well as 2,547 trios from family based studies, showed a definite relationship between several psychiatric disorders, including, for example, attention-deficiency hyperactivity disorder (ADHD) and panic disorder for Caucasian samples. ${ }^{42}$

We conclude that the tested polymorphisms, and in theory at least the COMT Val158Met polymorphism as described above, may have an influence on the prevalence of LID in PD, but this influence is small, and in our study confounded by a possible increased likelihood for PD to manifest itself, thus causing an increased disease duration in our patient population. In previous studies, we have observed that in both tardive dyskinesia as well as in LID, ${ }^{14,36,43}$ limbtruncal dyskinesia may have a genetic background other than orofacial dyskinesia. Unfortunately, the number of patients in this study with LID is relatively small $(\mathrm{N}=58)$, and too many suffered from both orofacial and limb-truncal dyskinesia to make further statistical analysis feasible.

The strengths of our study include the relatively large number of patients $(\mathrm{N}=232)$; the thorough assessment of the severity of dyskinesia by direct clinical assessment with AIMS; the application of Schooler and Kane's criteria to conclude to the presence of LID, and the assessment of seven putatively relevant polymorphisms of the COMT gene after carefully reviewing previous findings. The weaker points are the relatively low number of patients suffering from LID $(\mathrm{N}=58$, $25 \%$ ), and the co-occurrence of limb-truncal and orofacial dyskinesia in many of them. 
Table 1. SNPs associated with levodopa-induced dyskinesia

\begin{tabular}{lllc}
\hline SNP & Best model & OR $[95 \% \mathrm{CI}]$ & Permutation $p$-value \\
\hline rs165774 & Additive & $1.75[1.14-2.72]$ & 0.011 \\
rs4818 & Additive & $0.57[0.34-0.92]$ & 0.025 \\
rs4633 & Dominant & $0.45[0.23-0.89]$ & 0.027 \\
rs4680 & Dominant & $0.46[0.23-0.91]$ & 0.025
\end{tabular}

The results of this study illustrate that genetic variations of the activity of the COMT enzyme have only a modest influence on the clinical presentation of $\mathrm{PD}$, and its treatment complications such as LID. The vulnerability in PD apparently differs from that in other neuropsychiatric disorders, such as ADHD. ${ }^{42}$ It can been suggested that this may be related to low expression of DA-transporters within the prefrontal cortex and not within synapses, contrary to, for example, the striatum. ${ }^{19,22}$ It is possible that the abundance of DA-transporters within the striatum limits the influence of variations in COMT activity, because after re-uptake within dopaminergic terminals dopamine is generally processed differently (e.g., through mitochondrial monoamine oxidase). Moreover, the highly adaptive capacity of the functional nigrostriatal extrapyramidal connectivity may limit the influence of genetic variations in enzyme activity. This influence on the activity of the cortico-striatothalamo-cortical circuitry may be less than that within the prefrontal cortex or corticoid amygdala, which may play a role in causing other neuropsychiatric disorders. ${ }^{12}$ However, other PD manifestations, including cognitive symptoms, may be more dependent on the functioning of the prefrontal cortex. Recently, Zhang et al have demonstrated that COMT Val158Met polymorphism is probably not associated with increased risk of PD, but has an effect on the prefrontal executive function interacting with gender and dopaminergic medication. ${ }^{44}$ Specifically addressing manifestations related to dysfunction of the PFC (vigilance), corticoid amygdala (delusions) and hippocampal complex (hallucinations) may, in future, be more awarding when studying COMT gene polymorphisms.

\section{CONCLUSIONS}

The possible association between seven putatively relevant genetic variants of COMT gene and prevalence of LID in 232 Caucasian patients with PD was studied. Although the association of four polymorphisms reached statistical significance, the relationship was masked after discounting the time frame of the proportion of patients in the sample suffering from $P D$.

\section{ACKNOWLEDGMENTS}

This work resulted from a collaboration between the Mental Health Research Institute in Tomsk and the Groningen Research Institute of Pharmacy (GRIP) of the University of Groningen. The Russian part is carried out within the framework of Tomsk Polytechnic University Competitiveness Enhancement Program. The authors have no conflicts of interest to report.

\section{REFERENCES}

1. Birkmayer W, Hornykiewicz O. The L-3,4dioxyphenylalanine (DOPA)-effect in Parkinsonakinesia. Wien Klin Wochenschr. 1961;73:787-788.

2. Hornykiewicz O. A brief history of levodopa. $J$ Neurol. 2010;257(Suppl 2):S249-52.

3. Connolly BS, Lang AE. Pharmacological treatment of Parkinson disease: A review. JAMA. 2014;311(16):1670-1683.

4. Thanvi BR, Lo TC. Long term motor complications of levodopa: Clinical features, mechanisms, and management strategies. Postgrad Med J. 2004;80(946):452-458.

5. hanvi $\mathrm{B}$, Lo $\mathrm{N}$, Robinson $\mathrm{T}$. Levodopa-induced dyskinesia in Parkinson's disease: Clinical features, pathogenesis, prevention and treatment. Postgrad Med J. 2007;83(980):384-388.

6. Del Sorbo F, Albanese A. Levodopa-induced dyskinesias and their management. $J$ Neurol. 2008;255 Suppl 4:32-41.

7. Pilleri M, Antonini A. Therapeutic strategies to prevent and manage dyskinesias in Parkinson's disease. Expert Opin Drug Saf. 2015;14(2):281-294.

8. Rascol O, Brooks DJ, Korczyn AD, De Deyn PP, Clarke CE, Lang AE. A five-year study of the incidence of dyskinesia in patients with early Parkinson's disease who were treated with ropinirole or levodopa. N Engl J Med. 2000;342(20):14841491.

9. Huot P, Johnston TH, Koprich JB, Fox SH, Brotchie JM. The pharmacology of L-DOPA-induced dyskinesia in Parkinson's disease. Pharmacol Rev. 2013;65(1):171-222. 
10. Bargiotas $\mathrm{P}$, Konitsiotis S. Levodopa-induced dyskinesias in Parkinson's disease: Emerging treatments. Neuropsychiatr Dis Treat. 2013;9:16051617.

11. Cerasa A, Fasano A, Morgante F, Koch G, Quattrone A. Maladaptive plasticity in levodopainduced dyskinesias and tardive dyskinesias: Old and new insights on the effects of dopamine receptor pharmacology. Front Neurol. 2014;5:49.

12. Ivanova SA, Loonen AJ. Levodopa-induced dyskinesia is related to indirect pathway medium spiny neuron excitotoxicity: A hypothesis based on an unexpected finding. Parkinson Disease. 2016;2016:6461907.

13. Lohr JB, Kuczenski R, Niculescu AB. Oxidative mechanisms and tardive dyskinesia. CNS Drugs. 2003;17(1):47-62.

14. Al Hadithy AFY, Ivanova SA, Pechlivanoglou P, et al. Missense polymorphisms in three oxidativestress enzymes (GSTP1, SOD2, and GPX1) and dyskinesias in russian psychiatric inpatients from siberia. Hum Psychopharmacol. 2010;25(1):84-91.

15. Bonifacio MJ, Palma PN, Almeida L, Soares-daSilva P. Catechol-O-methyltransferase and its inhibitors in Parkinson's disease. CNS Drug Rev. 2007;13(3):352-379.

16. Guldberg HC, Marsden CA. Catechol-O-methyl transferase: Pharmacological aspects and physiological role. Pharmacol Rev. 1975;27(2):135206.

17. Craddock N, Owen MJ, O'Donovan MC. The catechol-O-methyl transferase (COMT) gene as a candidate for psychiatric phenotypes: Evidence and lessons. Mol Psychiatry. 2006;11(5):446-458.

18. Haavik J, Blau N, Thony B. Mutations in human monoamine-related neurotransmitter pathway genes. Hum Mutat. 2008;29(7):891-902.

19. Witte AV, Floel A. Effects of COMT polymorphisms on brain function and behavior in health and disease. Brain Res Bull. 2012;88(5):418428.

20. Lotta T, Vidgren J, Tilgmann C, et al. Kinetics of human soluble and membrane-bound catechol Omethyltransferase: A revised mechanism and description of the thermolabile variant of the enzyme. Biochemistry. 1995;34(13):4202-4210.

21. Ma Z, Liu H, Wu B. Structure-based drug design of catechol-O-methyltransferase inhibitors for CNS disorders. Br J Clin Pharmacol. 2014;77(3):410420.

22. Sesack SR, Hawrylak VA, Matus C, Guido MA, Levey AI. Dopamine axon varicosities in the prelimbic division of the rat prefrontal cortex exhibit sparse immunoreactivity for the dopamine transporter. J Neurosci. 1998;18(7):2697-2708.

23. Lee SG, Joo Y, Kim B, et al. Association of Ala72Ser polymorphism with COMT enzyme activity and the risk of schizophrenia in koreans. Hum Genet. 2005;116(4):319-328.

24. Nackley AG, Shabalina SA, Tchivileva IE, et al. Human catechol-O-methyltransferase haplotypes modulate protein expression by altering mRNA secondary structure. Science. 2006;314(5807):19301933.

25. $\mathrm{Xu} Z$, Zhang Z, Shi Y, et al. Influence and interaction of genetic polymorphisms in catecholamine neurotransmitter systems and early life stress on antidepressant drug response. $J$ Affect Disord. 2011;133(1-2):165-173.

26. Xiao Q, Qian Y, Liu J, Xu S, Yang X. Roles of functional catechol-O-methyltransferase genotypes in chinese patients with Parkinson's disease. Transl Neurodegener. 2017;6:11-017-0081-9. eCollection 2017.

27. Bialecka M, Kurzawski M, Klodowska-Duda G, Opala G, Tan EK, Drozdzik M. The association of functional catechol-O-methyltransferase haplotypes with risk of Parkinson's disease, levodopa treatment response, and complications. Pharmacogenet Genomics. 2008;18(9):815-821.

28. Meloto CB, Segall SK, Smith S, et al. COMT gene locus: New functional variants. Pain. 2015;156(10):2072-2083.

29. Higashiyama R, Ohnuma T, Takebayashi Y, et al. Association of copy number polymorphisms at the promoter and translated region of COMT with japanese patients with schizophrenia. Am J Med Genet B Neuropsychiatr Genet. 2016;171B(3):447457.

30. Seib C, Whiteside E, Voisey J, et al. Stress, COMT polymorphisms, and depressive symptoms in older australian women: An exploratory study. Genet Test Mol Biomarkers. 2016;20(8):478-481.

31. Ittiwut R, Listman JB, Ittiwut $\mathrm{C}$, et al. Association between polymorphisms in catechol-Omethyltransferase (COMT) and cocaine-induced paranoia in european-american and african-american populations. Am J Med Genet B Neuropsychiatr Genet. 2011;156B(6):651-660.

32. Loonen AJ, Doorschot $\mathrm{CH}$, van Hemert DA, Oostelbos MC, Sijben AE. The schedule for the assessment of drug-induced movement disorders (SADIMoD): Test-retest reliability and concurrent validity. Int $J$ Neuropsychopharmacol. 2000;3(4):285-296.

33. Loonen AJ, Doorschot $\mathrm{CH}$, van Hemert DA, Oostelbos MC, Sijben AE. The schedule for the assessment of drug-induced movement disorders (SADIMoD): Inter-rater reliability and construct validity. Int $J$ Neuropsychopharmacol. 2001;4(4):347-360.

34. Loonen AJ, van Praag HM. Measuring movement disorders in antipsychotic drug trials: The need to define a new standard. J Clin Psychopharmacol. 2007;27(5):423-430.

35. Schooler NR, Kane JM. Research diagnoses for tardive dyskinesia. Arch Gen Psychiatry. 1982;39(4):486-487.

36. Ivanova SA, Loonen AJ, Pechlivanoglou $\mathrm{P}$, et al. NMDA receptor genotypes associated with the vulnerability to develop dyskinesia. Transl Psychiatry. 2012;2:e67. 
37. Watanabe $M$, Harada $S$, Nakamura $T$, et al. Association between catechol-O-methyltransferase gene polymorphisms and wearing-off and dyskinesia in Parkinson's disease. Neuropsychobiology. 2003;48(4):190-193.

38. Contin M, Martinelli P, Mochi M, Riva R, Albani F, Baruzzi A. Genetic polymorphism of catechol-Omethyltransferase and levodopa pharmacokineticpharmacodynamic pattern in patients with Parkinson's disease. Mov Disord. 2005;20(6):734739.

39. Cheshire $\mathrm{P}$, Bertram K, Ling $\mathrm{H}$, et al. Influence of single nucleotide polymorphisms in COMT, MAO$\mathrm{A}$ and BDNF genes on dyskinesias and levodopa use in Parkinson's disease. Neurodegener Dis. 2014;13(1):24-28.

40. Ivanova SA, Alifirova VM, Zhukova IA, et al. Association of COMT gene polymorphisms with Parkinson's disease. Bulletin of Siberian Medicine. 2017;16(3):70-78.
41. de Lau LM, Verbaan D, Marinus J, Heutink P, van Hilten JJ. Catechol-O-methyltransferase Val158Met and the risk of dyskinesias in Parkinson's disease. Mov Disord. 2012;27(1):132-135.

42. Taylor S. Association between COMT Val158Met and psychiatric disorders: A comprehensive metaanalysis. Am J Med Genet B Neuropsychiatr Genet. 2018;177(2):199-210.

43. Al Hadithy AFY, Ivanova SA, Pechlivanoglou P, et al. Tardive dyskinesia and DRD3, HTR2A and HTR2C gene polymorphisms in russian psychiatric inpatients from siberia. Prog NeuroPsychopharmacol Biol Psychiatry. 2009;33(3):475481.

44. Zhang Y, Feng S, Nie K, et al. Catechol-Omethyltransferase Val158Met polymorphism influences prefrontal executive function in early Parkinson's disease. J Neurol Sci. 2016;369:347353. 